\title{
Stabilizing the Locomotor-Respiratory Coupling Using a Metronome to Save Energy
}

\author{
Charles P. Hoffmann* Sébastien J. Villard* Benoît G. Bardy* \\ (*) Movement to Health (M2H) Laboratory, EuroMov, Montpellier-1 University, France \\ E-mail: charles.hoffmann@univ-montp1.fr
}

\begin{abstract}
The Locomotor-Respiratory Coupling (LRC) is often evidenced by phase- or frequency-locking patterns. The model of the sine circle map is used here to characterize LRC. Several studies have suggested that a sound emitted by an external metronome can stabilize the LRC. Participants in our task were asked during a cycling exercise to synchronize either their respiration or their pedaling rate with an external auditory stimulus corresponding to their preferred respiratory and pedaling frequencies respectively. Our results showed a significant reduction in energy expenditure when participants breathed in sync with the auditory stimulation, but not accompanied by a change in the stabilization of LRC. A large within- as well as between-participants LRC variability, together with the spontaneous adoption of the most stable pace, contributes to explain this result.
\end{abstract}

\section{Introduction}

Many studies have reported a natural synchronization between respiration and locomotion in various species (fish, birds, mammals) and various forms of locomotion (running, cycling, rowing) [5]. Generally, entrainment between locomotion and respiration has been described by stable frequency mode-lockings such as the ones reported in humans: $1 / 1,1 / 2,1 / 3,2 / 3$, or $1 / 4$ [5]. The LocomotorRespiratory Coupling (LRC) is generally understood as originating from mechanical and neurological interactions. However, the LRC literature reveals several discrepancies related to the nature of the coupling, the factors that modulate it, and the concomitant energy expenditure.

\subsection{Link between expertise - LRC - energy}

Bramble \& Carrier [6] reported more entrainment between locomotion and respiration in experts than in novices. Other studies have shown more stable frequency locking in experts [1,9]. However, more than expertise in term of quantity of practice, the ability to coordinate locomotion and respiration seems to be related to the aerobic capacity of individuals [2, 4], a result demonstrating that the coupling between respiration and locomotion plays a role in the energy transfer. Bramble \& Carrier [5] suggested that a greater LRC could lead to a decrease in $\mathrm{O}_{2}$ consumption $\left(\mathrm{VO}_{2}\right)$. This idea was investigated by several authors and sometimes confirmed [3, 4, 11, 12]. However, Rassler \& Kohl [10] found no significant effect of LRC on energy consumption [10]. This discrepancy may be due to the differences in workload or populations between studies. Thus, the large differences in methodology used blur the current knowledge on LRC.

This study aimed to clarify the role and consequences of LRC stabilization on the energy expenditure during a sub-maximal exercise.

\subsection{Effect of an auditory stimulation}

In the motor control field, several studies have pointed out the anchoring effect, which captures the influence of an external stimulation on the coupling between two hands. Fink et al. [7] and others [6] have shown that a periodic auditory stimulation stabilizes both in-phase and anti-phase coordination (local stabilization) and postpones the transition from antiphase to in-phase (global stabilization). Haas et al. [8] reported a greater stabilization of the respiratory rhythm under a rhythmic external auditory stimulation. Moreover, they reported a greater stabilization when rhythmic tapping movements were added to the task. Several LRC studies found similar results and reported more entrainment between locomotion and respiration when a metronome was used to pace locomotion frequency [4]. So, an external stimulation seems to be a good mean to stabilize LRC.

This is an Open Access article distributed under the terms of the Creative Commons Attribution-Noncommercial License 3.0, which permits unrestricted use, distribution, and reproduction in any noncommercial medium, provided the original work is properly cited. 


\section{$1.3 \quad$ Objectives}

In this study, we proposed to consider LRC as the results of nonlinear-coupled oscillators and we use the sine circle map model to capture this phenomenon. We examined the stabilizing role of external periodic auditory information on LRC. We measured this effect by an increase in phase locking relationship between respiratory and locomotor cycles. Then, we proposed to confirm previous results showing that stronger LRC occurs with a decrease of oxygen consumption.

\section{Method}

\section{$2.1 \quad$ Subjects}

14 voluntary males athletes (22 to 32 years old) not specialized in endurance sports took part in the experiment. They all signed an informed consent before participating in the experiment, which was approved by the regional ethic review board (Comite Consultatif de Protection des Personnes de Nîmes SudMéditerranée 3).

\subsection{Experimental setup}

Experimental trials were performed on cycle ergometer (Ergoline 800, Hoechberg, Germany). Gaz exchanges and ventilation were recorded and analyzed continuously breath by breath by an automated system (ZAN 600 Ergo test, ZAN Messgerate $\mathrm{GmbH}$, Oberthulba, Germany). Breathing kinematics was measured by a thermistance placed inside the mask that participants wore during the experiment. The pedaling rhythm was calculated by an electromagnetic sensor placed on the left side of the ergometer, which collected the passage of a magnet fixed on the left crank of the ergometer. Both, temperature and electromagnetic signals were collected simultaneously at $200 \mathrm{~Hz}$ through a data acquisition board (NI USB6009, National Instruments, Austin, Texas, USA).

\subsection{Procedure}

Four sessions were involved. In the first session, participants completed a maximal incremental exercise $\left(\mathrm{VO}_{2}\right.$ max), in order to determine their individual power output at their anaerobic threshold $\left(P_{A T}\right)$. In the second session, after a 3-minutes warm-up, participants performed a 10-minutes exercise at $P_{A T}$ to determine their individual preferred respiratory $\left(R_{f} p r e f\right)$ and locomotor ( $L_{f}$ pref) frequencies. In the last two sessions, participants performed the same constant-load exercise at PAT divided in 5 minutes with acoustic stimulation (Sound) and 5 minutes without the stimulation (NoSound). In one of these two sessions, a periodic externally paced auditory sound was presented at $R_{f}$ pref to the participants who were instructed to exhale (RESP) in sync with the stimulation. In the other session, the acoustic stimulation was presented at $L_{f}$ pref and participants were requested to cycle (LOCO) in sync with the stimulation. Both, 5-minutes periods (Sound or NoSound) and the last two sessions (RESP or LOCO) were randomly distributed among participants.

\subsection{Data analysis}

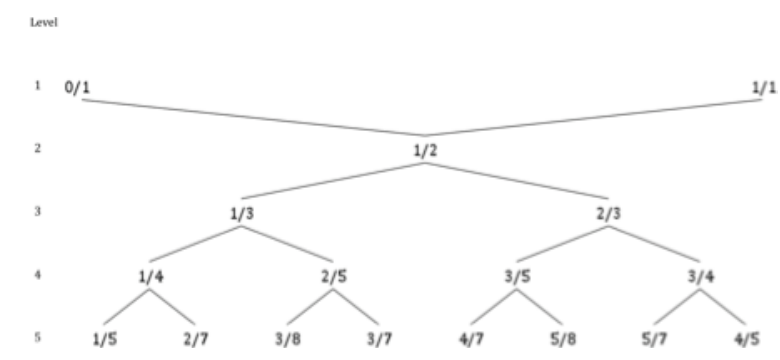

Fig 1: First five levels of the Farey Tree. This mathematical construction represents the hierarchical organization of frequency mode lockings predicted by the sine circle map model. Low order level presents more stable modes than high order ones. For instance, $1 / 2$ is predicted as more stable than $2 / 5$.

After having determined dates of expirations and pedaling strikes we calculated the frequency ratio for each respiratory cycle. We obtained a time-series of real quotients. We used the sine circle map to label the observed frequency ratio (e.g., 0.299) as a ratio of small integers (e.g., 1/3). The Farey tree (Fig 1) is a mathematical structure organizing the rational integer ratios by levels, where each level corresponds to the stability rank of each frequency ratio. For instance, 1/2 is predicted to be more stable than higher order frequency ratios such as $1 / 3$ or $2 / 3$. We obtained a distribution of Farey ratios. For each distribution, we determined the most frequent Farey ratio (i.e., the mode of the distribution) and calculated the dispersion of the relative phase between respiratory and pedaling cycles for this mode. The calculation of the relative phase was based on the equation used by McDermott, Van Emmerik \& Hamill [9] for 1/q ratios and modified to take into account $\mathrm{p} / \mathrm{q}$ ratios:

$$
R P_{i, R(p)}=\frac{t_{i, R(p)}}{T_{i}} \times 360^{\circ}
$$


where $\mathrm{RP}_{i, R(p)}$ represents the relative phase at the pedaling strike $i$ for a p/q mode locking, $t_{i, R(p)}$ represents the distance from the pedaling strike $i$ to the end of $\mathrm{p}$ respiratory cycles and $\mathrm{T}_{i}$ represents the period of the pedaling strike $i$. This measure of relative phase over time permit us to represent the relative phase dispersion by mapping each relative phase $i$ on the relative phase $\mathrm{i}+\mathrm{q}$ (Return Maps). As presented by McDermott et al. [9], we used the dispersion of relative phases around the identity line to assess the coupling strength.

We also used the percentage of occurrence of the frequency ratio mode, and a Phase Coupling index (PC) to test the effect of our experimental design on LRC. The PC index quantifies the variability of the relative phase on the return maps [14]. The perfect coupling (represent by $\mathrm{PC}=100 \%$ ) would represent all points of the return maps lying on the identity line.

In conclusion, to assess the stability of the LRC, three variables were computed, i.e., (i) the most frequent Farey ratio (i.e., the mode of the distribution), (ii) the dispersion of the relative phase between respiratory and pedaling cycles for this mode, and (iii) the mean number of consecutive cycles spent on the modal frequency ratio.

\subsection{Statistical analysis}

A repeated-measures ANOVA was performed to assess the effect of the auditory stimulation (SOUND vs. NOSOUND) on the LRC and the concomitant energy expenditure in both conditions (LOCO vs. RESP). Post hoc Scheffe tests were conducted to determine which conditions were significantly different. The level of significance was set at alpha lower than 0.05 for all tests.

\section{Results}

The auditory stimulation produced a significant decrease in the oxygen consumption $(\mathrm{t}(13)=2.47, \mathrm{p}<$ $.05)$ when participants breathed in sync with the external sound (Fig 2). However, the auditory stimulus had no significant effect on the percentage of occurrence of the frequency ratio mode, the dispersion of the relative phase between respiratory and pedaling cycles for the mode, the mean number of consecutive cycles spent on the modal frequency ratio.

\section{Energy expenditure}

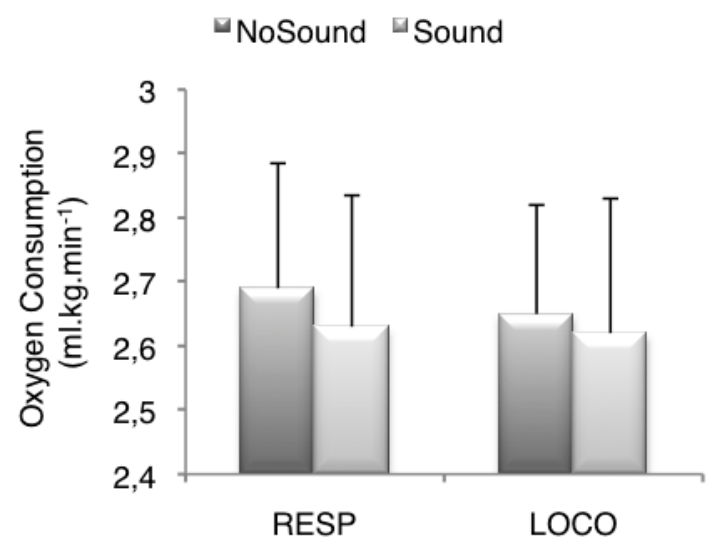

Fig 2. Oxygen consumption comparing NoSound condition (black) and Sound condition (grey) for both RESP and LOCO conditions.

A. Rapports de Fréquence
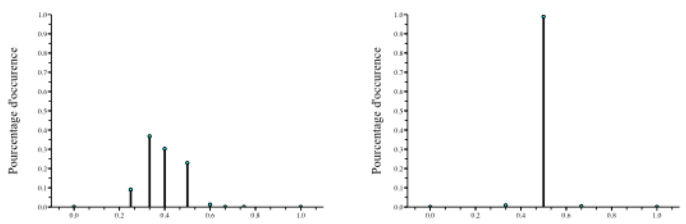

B. Phases Relatives
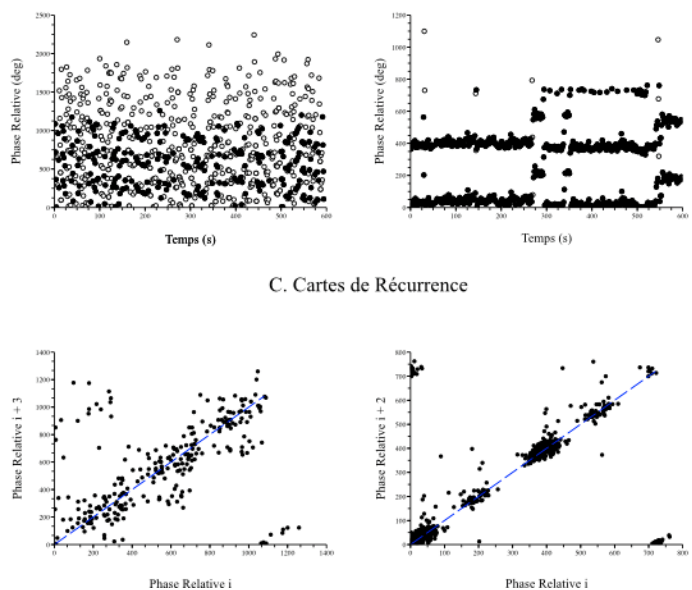

Cartes de Récurrence

Fig 3: LRC in two participants. The first two panels (A. Frequency Ratios Distribution) represent the distribution of the frequency ratios. The next two panels (B. Relative Phases) represent 3 minutes of relative phase. The last four panels (C. Return Maps) represent the dispersion of the relative phase in return maps. 


\section{Discussion}

Until now, no studies were dedicated specifically to the potential effect of an external stimulation on energy expenditure through LRC stabilization. In our study, we found a significant reduction of the oxygen consumption when participants were instructed to breath in sync with the auditory stimulation. However, this reduction was not accompanied by a stabilization of the LRC. One explanation is the important variability observed within- as well as betweenparticipants (Fig 3), revealing some strong but contradictory effects. Another explanation is the fact that our participants were already in their preferred and stable frequency ratio. These results need to be confirmed. However, our method to assess frequency locking and relative phase dispersion in LRC allows to assess a behavioural invariant - the frequency ratio organized hierarchically in a Farey tree, and an index of variability, i.e., the phase coupling.

\section{Conclusion}

These experimental results and the previous findings obtained in the LRC literature point out the difficulty to capture LRC. Here, we have used a method previously explored by McDermott et al. [9], which uses the complex frequency mode locking associated with the relative phase dispersion to express the coupling strength between locomotion and respiration. LRC is a universal phenomenon underlying the supply and production of energy. Rhythmic activities such as walking, running, swimming, rowing are all exhibiting LRC. In our study we have not found a consistent stabilizing effect of an auditory stimulation on LRC stability, in spite of an interesting decrease in oxygen consumption.

\section{References}

[1] P. G. Amazeen, E. L. Amazeen, and P. J. Beek. Coupling of breathing and movement during man- ual wheelchair propulsion. J Exp Psychol Hum Percept Perform, 27(5):1243-59, 2001.
[2] P. Bernasconi, P. Burki, A. Buhrer, E. A. Koller, and J. Kohl. Running training and co-ordination between breathing and running rhythms during aerobic and anaerobic conditions in humans. Eur J Appl Physiol, 70(5):387-93, 1995.

[3] P. Bernasconi and J. Kohl. Analysis of coordination between breathing and exercise rhythms in man. J Physiol, 471:693-706, 1993.

[4] M. R. Bonsignore, G. Morici, P. Abate, S. Ro- mano, and G. Bonsignore. Ventilation and entrain- ment of breathing during cycling and running in triathletes. Med Sci Sports Exerc, 30(2):239-45, 1998.

[5] D. M. Bramble and D. R. Carrier. Running and breathing in mammals. Science, 219(4582):251-6, 1983.

[6] W. D. Byblow, R. G. Carson, and D. Goodman. Expressions of asymmetries and anchoring in bi- manual coordination. Hum Mov Sci, 13(1):3-28, February 1994.

[7] P. W. Fink, P. Foo, V. K. Jirsa, and J. A. Kelso. Local and global stabilization of coordination by sensory information. Exp Brain Res, 134(1):9-20, Sep 2000.

[8] F. Haas, S. Distenfeld, and K. Axen. Effects of perceived musical rhythm on respiratory pattern. $J$ Appl Physiol, 61(3):1185-1191, Sep 1986.

[9] W. J. McDermott, R. E. Van Emmerik, and J. Hamill. Running training and adaptive strate- gies of locomotorrespiratory coordination. Eur J Appl Physiol, 89(5):43544, 2003.

[10] B. Rassler and J. Kohl. Analysis of coordina- tion between breathing and walking rhythms in humans. Respir Physiol, 106(3):317-27, 1996.

[11] B. Rassler and J. Kohl. Coordination-related changes in the rhythms of breathing and walk- ing in humans. Eur $J$ Appl Physiol, 82(4):280-8, 2000.

[12] S. Villard, J.-F. Casties, and D. Mottet. Dynamic stability of locomotor respiratory coupling during cycling in humans. Neurosci Lett, 383(3):333-338, Aug 2005. 